\title{
The impact of visit-to-visit variability in blood pressure on renal function
}

\author{
Tatsuo Kawai, Mitsuru Ohishi, Kei Kamide, Miyuki Onishi, Yasushi Takeya, Yuji Tatara, Ryosuke Oguro, \\ Koichi Yamamoto, Ken Sugimoto and Hiromi Rakugi
}

Hypertension is an important risk factor for cardiovascular diseases such as chronic kidney disease. It is still not fully understood how blood pressure impacts the kidneys. In this study, we aimed to establish the significance of visit-to-visit variability in blood pressure for renal function. We analyzed 143 consecutive patients undergoing renal Doppler ultrasonography in our hospital ward and measured blood pressure at outpatient visits six or more times. We analyzed the correlation between visit-to-visit variability in blood pressure and multiple clinical parameters, including albuminuria and resistive index evaluated by renal Doppler ultrasonography, which is thought to be a good indicator of renal vascular resistance. Subjects with higher variability in systolic blood pressure showed a significantly higher prevalence rate of clinical albuminuria and microalbuminuria, and showed significantly higher resistive index. Stepwise multiple regression analysis showed that variability in systolic blood pressure was a significant risk factor for higher resistive index, independent of other renal risk factors. Visit-to-visit variability in blood pressure correlates significantly with renal function and renal arteriosclerotic change. This parameter could provide additional information about renal arteriosclerotic change independent of estimated glomerular filtration rate and albuminuria, and should be considered a therapeutic target for renal protection.

Hypertension Research (2012) 35, 239-243; doi:10.1038/hr.2011.170; published online 20 October 2011

Keywords: blood pressure; renal Doppler ultrasonography; renal vascular resistance; resistive index; visit-to-visit variability

\section{INTRODUCTION}

Hypertension is one of the most important treatable risk factors for chronic kidney disease (CKD). ${ }^{1-2}$ However, the mechanism by which hypertension influences renal function and produces renal vascular damage is still incompletely understood. Blood pressure (BP) level is widely recognized to strongly correlate with $\mathrm{CKD}$, as it does with other cardiovascular diseases. ${ }^{3-5}$ Clinical guidelines recommend using mean BP, systolic BP (SBP) and diastolic BP (DBP) for diagnosis and management of hypertension to prevent cardiovascular events such as progression of renal dysfunction.

Recently, additional parameters, such as variability in BP, were demonstrated to correlate with cardiovascular events. ${ }^{6}$ For example, Rothwell et al. ${ }^{7}$ showed that visit-to-visit variability in SBP is a strong predictor of stroke. There are no data available regarding the relationship between renal function and visit-to-visit variability in BP.

The resistive index (RI: (peak systolic velocity-end diastolic velocity)/peak systolic velocity at segmental arteries in kidney) evaluated by renal Doppler ultrasonography (RDU) is considered a useful index of renal vascular resistance secondary to arteriosclerosis ${ }^{8-10}$ and is a good prognostic indicator of renal function. ${ }^{11-16}$ We previously reported that the RI might be a more efficacious parameter for the evaluation of very early renal damage than estimated glomerular filtration rate (eGFR). ${ }^{17}$ It is thought that renal vascular damage caused by atherosclerotic risk factors such as hypertension and variability of BP could be evaluated more precisely by the RI.

In this study, we assessed the hypothesis that visit-to-visit variability in BP correlates significantly with renal function. We investigated the correlation between visit-to-visit variability in BP (expressed as standard deviation (s.d.) and coefficient variant (CV: s.d./mean)) and various renal function parameters such as eGFR, RI and level of proteinuria. We also investigated the correlation between visit-to-visit variability in $\mathrm{BP}$ and other biochemical parameters.

\section{METHODS}

\section{Study subjects}

Figure 1 shows a flow chart of the selection process for study participants. In our hospital ward, almost all admitted patients for several internal diseases, such as diabetes mellitus, hypertension, $\mathrm{CKD}$ and so on, undergo RDU to evaluate renal arteriosclerotic change. We initially enrolled 281 consecutive patients with and without CKD undergoing RDU in our hospital ward between February 2009 and May 2011. Patients were excluded if they had renal artery stenosis $(n=16)$, renal transplant $(n=1)$ or were on dialysis $(n=1)$. Of the 263 patients, 120 returned for follow-up visit with BP measurement less than six times; therefore, a total of 143 patients were included in this study. In addition, 


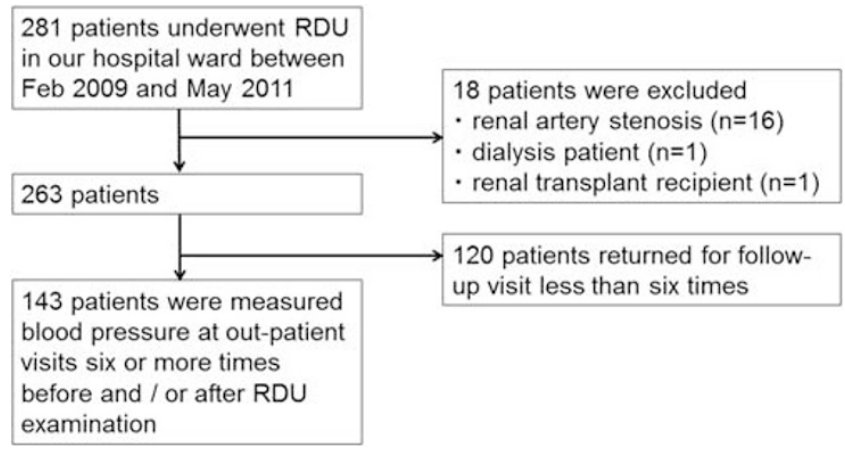

Figure 1 Flow chart of the selection process of study subjects.

we analyzed a subgroup of 104 patients who had BP measured at outpatient visits 10 or more times before and/or after RDU examination.

Visit-to-visit variability in BP was defined as the s.d. and CV in BP. Variables were calculated from BP measured at six serial visits before and/or after RDU examination.

Subjects underwent biochemical examination of the blood and urine. Clinical parameters considered in this study were: height, weight, body mass index, eGFR, serum lipid profile, fasting blood glucose, glycosylated hemoglobin, hemoglobin Alc, additional biochemical parameters, proteinuria level, SBP and DBP at the time of RDU, smoking history and drug profile. The Clinical Investigations Ethics committee of Osaka University Hospital approved the study protocol. The study was performed in adherence with the principles of the Declaration of Helsinki and according to Good Clinical Practice standards.

\section{Ultrasonographic determination \\ RI was calculated as: \\ $\mathrm{RI}=$ (peak systolic velocity-end diastolic velocity)/peak systolic velocity.}

Patients were placed in a supine position, and the size of the left and right kidneys and the flow velocity in the aorta and renal arteries were evaluated to detect morphological abnormality or renal artery stenosis. RI was determined in three different segmental arteries of both kidneys, and expressed as the mean of these values; this method was reported to be identical and technically easy to perform so that reproducibility of RI could be improved. ${ }^{18-20}$ Previous studies indicated that reliable RI measurements depended on proper measuring techniques performed by experienced operators, ${ }^{21}$ so in this study Doppler examinations were performed by the three experienced operators (TK, KK and MO) using a XARIO SSA-660A ultrasound machine (TOSHIBA, Tokyo, Japan) with a $2.5-\mathrm{MHz}$ sector transducer

\section{Renal function}

eGFR was calculated using the following equation:

eGFR $\left(\mathrm{ml} \mathrm{min}^{-1}\right.$ per $\left.1.73 \mathrm{~m}^{2}\right)=194 \times$ creatinine $-1.094 \times$ age $-0.287(\times 0.739$ if female). ${ }^{22}$

The level of albuminuria was evaluated according to the American Diabetes Association classification. ${ }^{23}$ The albumin/creatinine ratio in spot urine was used to classify proteinuria as follows: no proteinuria: $<30 \mathrm{mg} \mathrm{g}^{-1}$ creatinine; microalbuminuria: $\quad 30-300 \mathrm{mg} \mathrm{g}^{-1}$ creatinine; clinical albuminuria: $\geq 300 \mathrm{mg} \mathrm{g}^{-1}$ creatinine.

\section{BP measurements}

Conventional BP was measured by trained observers with an electronic sphygmomanometer (HEM-705IT or HEM-711; OMRON, Kyoto, Japan). Following the guidelines for the management of hypertension, at every visit (monthly-bimonthly), clinic BP was measured at least two times in sitting position after $5 \mathrm{~min}$ rest, and we adopted the average of two readings as office $\mathrm{BP}$ if the difference of measured values was $<5 \mathrm{~mm} \mathrm{Hg}$. When the difference of measured values was more than $5 \mathrm{~mm} \mathrm{Hg}$, additional measurements were conducted to obtain stable $\mathrm{BP}$ readings and we adopted the average of the two stable readings as office $\mathrm{BP}$.

\section{Statistical analysis}

All data are expressed as mean \pm s.d. Differences between groups were analyzed employing the unpaired Student's $t$-test and Pearson's $\chi^{2}$ test. Multiple linear regression analysis was performed to determine more related variables for RI. Stepwise multiple regression analysis was used to identify possible determinants of RI. The level of significance was defined as $P<0.05$. All statistical analyses were performed using JMP (JMP version 8.0.1, Cary, NC, USA).

\section{RESULTS}

This study included 143 consecutive patients who underwent RDU in our hospital ward from February 2009 to May 2011 and who had their BP measured at outpatient visits six or more times before and/or after RDU examination (Figure 1).

Table 1 reports baseline patient characteristics. Mean age was $68.1 \pm 13.0$ years. In all, 79 patients were women and 64 were men. A total of 133 patients $(93.0 \%)$ were receiving treatment with antihypertensive agents, including angiotensin receptor blockers, angiotensin-converting enzymes inhibitors, calcium channel blockers, diuretics, $\beta$-blockers, $\alpha$-blockers or aldosterone blockers. In all, 95 patients $(66.4 \%)$ were receiving two or more different antihypertensive agents; 58 patients $(40.8 \%)$ were taking statins for dyslipidemia; and 51 patients $(35.7 \%)$ had type 2 diabetes mellitus. Table 2 showed comparison of baseline clinical characteristic of the subjects between lower s.d. in SBP group $(N=71$; mean \pm s.e.m.: $8.91 \pm 0.26)$ and higher s.d. in SBP group ( $N=72$; mean \pm s.e.m.: $17.49 \pm 0.58)$. Higher s.d. in SBP group showed significantly higher RI, lower DBP and higher prevalence rate of type 2 diabetes mellitus.

Firstly, we investigated how multiple clinical parameters correlated with variability in BP. Subjects with diabetes mellitus (DM) showed a significantly higher variability in SBP than those without DM (s.d. in SBP, patients with DM: $14.46 \pm 5.97$ vs. patients without DM: $12.32 \pm 5.68, P<0.05 ; \mathrm{CV}$ in SBP, patients with DM: $0.10 \pm 0.04$ vs. patients without $\mathrm{DM}: 0.09 \pm 0.04, P<0.05)$. There were no significant correlations between variability in BP and usage of each antihypertensive agent (angiotensin receptor blockers, angiotensinconverting enzymes inhibitors, calcium channel blocker, diuretics, $\beta$ blockers, $\alpha$-blockers or aldosterone blockers).

Secondly, we compared RI, eGFR and level of proteinuria between the quartile of s.d. in SBP and CV in SBP to investigate how variability of BP correlated with renal function. Patients with higher variability in SBP showed a significantly higher prevalence rate of clinical albuminuria and microalbuminuria (Pearson's $\chi^{2}$ test, $P=0.0014$ ) (Figure 2) and a significantly higher RI (Figure 3). However, there was no significant correlation between variability in SBP and eGFR.

As Rothwell et al. ${ }^{7}$ suggested that risks of stroke and coronary events increased in relation to maximum SBP in outpatient visits, we investigated the correlation between maximum SBP in six outpatients visit and RI, eGFR and proteinuria. RI was significantly correlated with maximum SBP $(R=0.179, P<0.05)$; however, eGFR was not significantly correlated with maximum SBP $(R=0.080, P=0.344)$. Patients with albuminuria or microalbuminuria showed significantly higher maximum SBP (patients with albuminuria or microalbuminuria: $167.5 \pm 25.0$ vs. patients without albuminuria: $154.6 \pm 15.8$, $P<0.005$.)

In the recent article from the NHANES III, BP measured at outpatient visits for three times were analyzed as BP variability, and higher levels of short-term visit-to-visit SBP variability were associated with increased all-cause mortality. ${ }^{24}$ Therefore, to further investigate, 
Table 1 Baseline clinical characteristic of all subjects

\begin{tabular}{lr}
\hline Men/women & $64 / 79$ \\
Age (years) & $68.1 \pm 13.0$ \\
eGFR $\left(\mathrm{ml} \mathrm{min}^{-1}\right.$ per $\left.1.73 \mathrm{~m}^{2}\right)$ & $59.0 \pm 24.0$ \\
Resistive index & $0.70 \pm 0.08$ \\
Systolic blood pressure $(\mathrm{mm} \mathrm{Hg})$ & $138.2 \pm 22.3$ \\
Diastolic blood pressure $(\mathrm{mm} \mathrm{Hg})$ & $77.4 \pm 14.2$ \\
Treated with antihypertensive agents & $133(93.0 \%)$ \\
Treated with statins & $58(40.8 \%)$ \\
Subjects with type 2 diabetes mellitus & $51(35.7 \%)$
\end{tabular}

Abbreviations: eGFR, estimated glomerular filtration rate. Values are expressed as the mean \pm s.d. (range) or numbers.

Table 2 Comparison of baseline clinical characteristic of the subjects between lower s.d. in SBP group ( $N=71$; mean \pm s.e.m.:

$8.91 \pm 0.26)$ and higher s.d. in SBP group ( $N=72$; mean \pm s.e.m.: $17.49 \pm 0.58$

\begin{tabular}{lccr}
\hline & \multicolumn{2}{c}{ Variability in SBP (s.d.) } & \\
\cline { 2 - 3 } & Lower group & Higher group & P-value \\
\cline { 2 - 3 } & $32 / 39$ & $32 / 40$ & 0.940 \\
Men/women & $67.6 \pm 13.0$ & $68.6 \pm 13.1$ & 0.323 \\
Age (years) & $60.6 \pm 25.1$ & $57.5 \pm 22.9$ & 0.224 \\
eGFR (ml min ${ }^{-1}$ per 1.73 m $^{2}$ ) & $0.68 \pm 0.07$ & $0.72 \pm 0.08$ & $<0.005$ \\
Resistive index & $137.9 \pm 22.2$ & $138.5 \pm 22.7$ & 0.438 \\
Systolic blood pressure (mm Hg) & $79.8 \pm 14.9$ & $75.1 \pm 13.1$ & $<0.05$ \\
DBP (mm Hg) & $65(91.5 \%)$ & $68(95.8 \%)$ & 0.302 \\
Treated with antihypertensive agents & $27(38.0 \%)$ & $31(43.7 \%)$ & 0.495 \\
Treated with statins & $20(31.7 \%)$ & $31(51.7 \%)$ & $<0.05$ \\
Subjects with type 2 diabetes mellitus & 20.05
\end{tabular}

Abbreviations: DBP, diastolic blood pressure; eGFR, estimated glomerular filtration rate; SBP, systolic blood pressure.

Values are expressed as the mean \pm s.d. (range) or numbers. Higher s.d. in SBP group showed significantly higher resistive index, lower DBP and higher prevalence rate of type 2 diabetes mellitus.

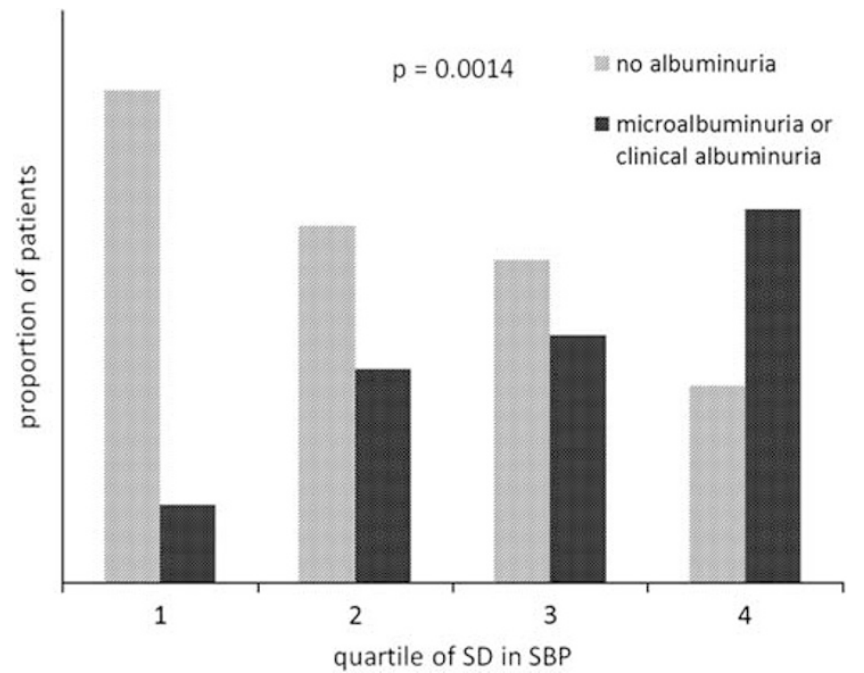

Figure 2 Association between s.d. quartile in SBP and proteinuria. Patients with higher s.d. in SBP showed a higher prevalence rate of microalbuminuria and clinical albuminuria (Pearson's $\chi^{2}$ test, $P=0.0014$ ).

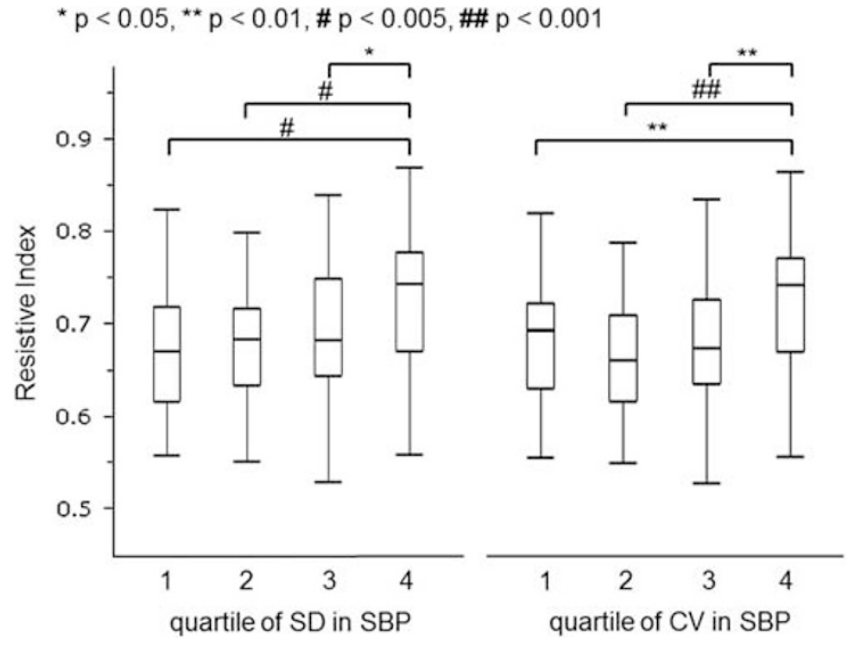

Figure 3 Association between the resistive index and variability in blood pressure analyzed in 143 patients with blood pressure measured at least six times at outpatient visits. Left: association between the resistive index and quartile of s.d. in SBP. Quartile 1: 0.68 \pm 0.01 ; quartile 2: $0.69 \pm 0.01$; quartile 3: $0.70 \pm 0.01$; and quartile 4: $0.73 \pm 0.01$ (mean \pm s.e.m.). Right: Association between the resistive index and quartile of CV in SBP. Quartile 1 : $0.69 \pm 0.01$; quartile 2: $0.68 \pm 0.01$; quartile 3 : $0.69 \pm 0.01$; and quartile 4: $0.74 \pm 0.01$ (mean \pm s.e.m.). Patients with higher variability in SBP showed a significantly higher RI than patients with lower variability.

we analyzed a total of 171 patients with BP measured at outpatient visits three or more times before and/or after RDU examination and calculated s.d. and CV similarly. Subjects with higher variability in SBP from three BP measurements also showed a significantly higher RI with lower decision correlation (Figure 4). On the other hand, patients with higher variability in SBP from three BP measurements showed the tendency of higher prevalence rate of clinical albuminuria and microalbuminuria, but there was no significant correlation (data not shown).

Multivariate analysis revealed that s.d. in SBP and CV in SBP was significant risk factors for higher RI independent of other renal risk factors. Table 3 showed that correlation between RI and variability in SBP. Without adjustment, s.d. in SBP and CV in SBP were significantly correlated with RI (s.d. in SBP: $P=0.0006$; $C V$ in SBP: $P=0.0020$ ). In model 1, adjusted for age, body mass index, SBP, eGFR and with or without DM, s.d. in SBP and CV in SBP were also significantly correlated with RI (s.d. in SBP: $P<0.0001$; CV in SBP: $P<0.0001$ ). In model 2, adjusted for model 1+with or without smoking, s.d. in SBP and CV in SBP were significantly correlated with RI too (s.d. in SBP: $P<0.0001$; CV in SBP: $P<0.0001$ ). In model 3, adjusted for model $1+$ with or without albuminuria, s.d. in SBP and CV in SBP were also significantly correlated with RI (s.d. in SBP: $P<0.0001$; CV in SBP: $P=0.0002$ ).

\section{DISCUSSION}

This is the first report analyzing the impact of variability in BP on renal function. We showed that visit-to-visit variability in BP was significantly correlated with the level of albuminuria and was a risk factor for higher RI independent of other traditional renal risk factors.

Previous studies revealed that the RI was a useful predictor of renal dysfunctions ${ }^{11-16}$ and correlated significantly with organ damage. Measurement of RI in addition to low-grade albuminuria is reportedly useful for target organ damage screening in patients with resistant 

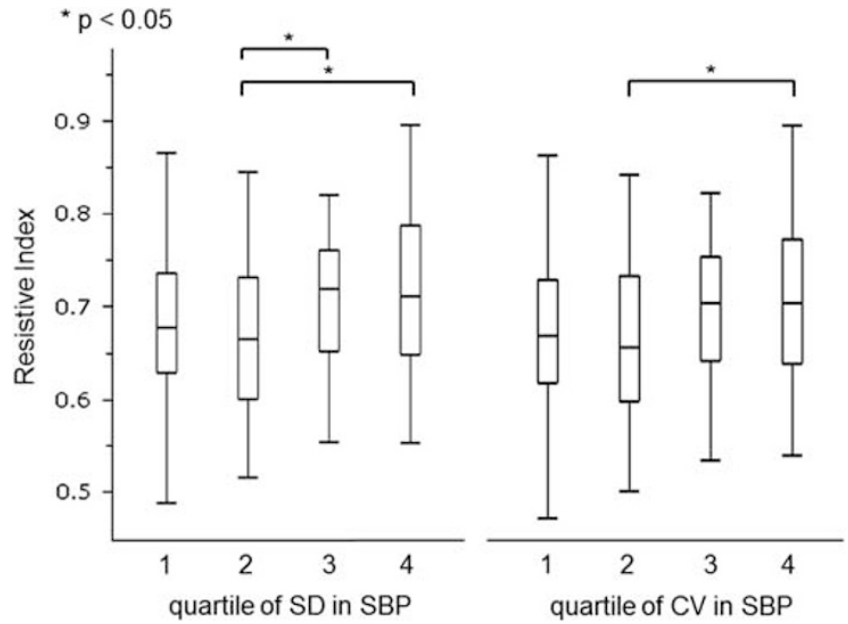

Figure 4 Association between the resistive index (RI) and variability in blood pressure analyzed with 171 patients with s.d. and CV of three times blood pressure measurement at outpatient visits. Left: association between the RI and quartile of s.d. in SBP. Patients with quartile 1: $0.68 \pm 0.01$; quartile 2: $0.67 \pm 0.01 ;$ quartile $3: \quad 0.71 \pm 0.01 ;$ and quartile $4: 0.72 \pm 0.01$ (mean \pm s.e.m.). Right: association between the RI and quartile of $\mathrm{CV}$ in SBP. Patients with quartile 1: $0.69 \pm 0.01$; quartile 2: $0.68 \pm 0.01$; quartile 3: $0.70 \pm 0.01$; and quartile 4: $0.72 \pm 0.01$ (mean \pm s.e.m.). Patients with higher variability in SBP showed significant higher RI than patients with lower variability.

hypertension. ${ }^{10}$ RI values were independently correlated with carotid intima-media thickness in patients with never-treated essential hypertension $^{9}$ and metabolic syndrome. ${ }^{8}$ These results suggest that renal vascular resistance indicated by the RI reflects the degree of systemic atherosclerosis, and that RI can serve as a useful marker to detect and evaluate atherosclerotic diseases due to cardiovascular diseases risk factors, such as hypertension, DM, dyslipidemia and metabolic syndrome.

Previously, a very interesting investigation was reported concerning the relationship between RI and histopathological analysis by renal biopsy, including glomerular sclerosis, interstitial fibrosis/tubular atrophy, interstitial infiltration and arteriolosclerosis. By stepwise multiple regression analysis, only arteriolosclerosis was found to be an independent risk factor for increased RI. ${ }^{25}$ Therefore, the results of our study showing the correlation between visit-to-visit variability in $\mathrm{BP}$ and RI indicate that visit-to-visit variability in SBP is an independent risk factor for renal arteriosclerotic change, and could be a useful predictor of renal dysfunction.

Multivariate analysis revealed that variability in SBP was significant risk factors for higher RI independent of not only other renal risk factors, but also eGFR and albuminuria. It indicated that employing variability in SBP in addition to eGFR and albuminuria to evaluate renal arteriosclerotic change is convenient and efficacious in clinical practice.

Previous reports suggest that DBP variability is independently predictive of nephropathy, but not of retinopathy, in DM patients. ${ }^{26}$ We found very similar results in hypertensive patients. In this study, variability in $\mathrm{BP}$ correlated significantly with the level of proteinuria and RI, but not with eGFR; although eGFR calculated easily from serum creatinine level, age and sex is useful for renal function screening, it is generally difficult to assess the pathogenesis of CKD using only eGFR. We previously reported that the RI might be a more sensitive parameter for the evaluation of very early renal vascular damage than eGFR, ${ }^{17}$ so we thought that RI could detect
Table 3 Correlation between variability in SBP and RI

\begin{tabular}{|c|c|c|}
\hline & \multicolumn{2}{|c|}{ P-value } \\
\hline & $S D$ in $S B P$ & $C V$ in $S B P$ \\
\hline Not adjusted & 0.0006 & 0.0020 \\
\hline \multicolumn{3}{|l|}{ Model 1} \\
\hline $\begin{array}{l}{ }^{*} \text { Adjusted for age, BMI, SBP, eGFR and } \\
\text { with or without DM }\end{array}$ & $<0.0001$ & $<0.0001$ \\
\hline \multicolumn{3}{|l|}{ Model 2} \\
\hline $\begin{array}{l}{ }^{*} \text { Adjusted for model } 1+\text { with or without } \\
\text { smoking }\end{array}$ & $<0.0001$ & $<0.0001$ \\
\hline \multicolumn{3}{|l|}{ Model 3} \\
\hline $\begin{array}{l}\text { *Adjusted for model } 1+\text { with or without } \\
\text { albuminuria }\end{array}$ & $<0.0001$ & 0.0002 \\
\hline
\end{tabular}

Abbreviations: BMI, body mass index; CV, coefficient of variation; DM, diabetes mellitus; eGFR, estimated glomerular filtration rate; RI, resistive index; SBP, systolic blood pressure. Without adjustment model, model 1 (adjusted for age, BMI, SBP, eGFR and with or without DM), model 2 (adjusted for model 1 +with or without smoking) and model 3 (adjusted for model $1+$ with or without albuminuria). In every model, s.d. in SBP and CV in SBP is significantly correlated with RI.

early renal damage correlated with variability in BP more sensitively than eGFR, and that this is the reason why higher variability in SBP was significantly correlated with higher RI and higher prevalence rate of clinical albuminuria and microalbuminuria, but not with eGFR.

Based on previous large clinical trials, mean BP is widely recognized to correlate strongly with cardiovascular diseases, including CKD, and clinical guidelines recommend using mean BP for diagnosis and management of hypertension to prevent cardiovascular event. This study suggests that visit-to-visit variability in BP should be considered a new therapeutic target for renal protection independent of mean BP.

Rothwell et al. ${ }^{27}$ reported that calcium channel blocker and $\beta$ blockers had different effects on variability of BP. In this study, however, there were no differences according to the type of antihypertensive agent (angiotensin receptor blockers, angiotensin-converting enzymes inhibitors, calcium channel blocker, diuretics, $\beta$-blockers, $\alpha$ blockers or aldosterone blockers). One explanation for this is that most of our subjects were receiving two or more different antihypertensive agents. Therefore, studies comparing each antihypertensive agent independently are necessary to evaluate their renoprotective effects.

Compared with lower s.d. in SBP group, higher s.d. in SBP group showed significantly higher RI, lower DBP and higher prevalence rate of type $2 \mathrm{DM}$. With aging or atherosclerotic disease, the arterial wall is generally stiffening, and the elasticity reduces; thus, DBP lowers and the pulse pressure rises. ${ }^{28-29}$ The decrease in DBP is thought to reflect vascular damage and increased risk of atherosclerosis. Therefore, the vascular damage induced by cardiovascular risk factors such as DM and hypertension is thought to be correlated with variability in BP.

We showed that subjects with higher variability in SBP from three BP measurements also showed a significantly higher RI with lower decision correlation. In clinical setting, the BP variability calculated from few outpatient visits is thought to be more convenient and easy-to-use index to assess the arteriosclerotic change of patients, although for more accurate assessments we could take more BP measurements in account.

\section{Study limitations}

This study has several limitations. First, our study was observational and cross-sectional. Longitudinal, prospective studies are necessary to evaluate the utility of measuring variability in BP for predicting the 
progression of atherosclerotic diseases and renal prognosis. Second, our sample size was relatively small. Third, because patients were recruited at the university hospital in this study, most patients had already been under medical treatment for hypertension, dyslipidemia and diabetes at the time of investigation; therefore, several parameters such as BP, lipid profile and glycosylated hemoglobin, hemoglobin Alc might have been influenced by medical treatment.

\section{Conclusion}

In conclusion, this study documents the impact of variability in BP on renal function. Visit-to-visit variability in BP is correlated significantly with renal function evaluated by the RI and albuminuria. This parameter could be a useful predictor of renal dysfunction and should be considered as a therapeutic target for renal protection.

\section{CONFLICT OF INTEREST}

The authors declare no conflict of interest.

\section{ACKNOWLEDGEMENTS}

This study was funded by a Grant-in-Aid (No. 22510211) from the Ministry of Education, Science and Culture and the NOVARTIS Foundation for Gerontological Research.

1 Shulman NB, Ford CE, Hall WD, Blaufox MD, Simon D, Langford HG, Schneider KA. Prognostic value of serum creatinine and effect of treatment of hypertension on renal function. Results from the hypertension detection and follow-up program. The Hypertension Detection and Follow-up Program Cooperative Group. Hypertension 1989; 13(Suppl): 180-193.

2 Walker WG, Neaton JD, Cutler JA, Neuwirth R, Cohen JD. Renal function change in hypertensive members of the Multiple Risk Factor Intervention Trial. Racial and treatment effects. The MRFIT Research Group. JAMA 1992; 268: 3085-3091.

3 Klag MJ, Whelton PK, Randall BL, Neaton JD, Brancati FL, Ford CE, Shulman NB, Stamler J. Blood pressure and end-stage renal disease in men. N Engl J Med 1996; 334: 13-18.

4 Klag MJ, Whelton PK, Randall BL, Neaton JD, Brancati FL, Stamler J. End-stage renal disease in African-American and white men. 16-year MRFIT findings. JAMA 1997; 277: 1293-1298.

5 Peterson JC, Adler S, Burkart JM, Greene T, Hebert LA, Hunsicker LG, King AJ, Klahr S, Massry SG, Seifter JL. Blood pressure control, proteinuria, and the progression of renal disease. The Modification of Diet in Renal Disease Study. Ann Intern Med 1995; 123 754-762.

6 Rothwell PM. Limitations of the usual blood-pressure hypothesis and importance of variability, instability, and episodic hypertension. Lancet 2010; 375: 938-948.

7 Rothwell PM, Howard SC, Dolan E, O'Brien E, Dobson JE, Dahlof B, Sever PS, Poulter NR. Prognostic significance of visit-to-visit variability, maximum systolic blood pressure, and episodic hypertension. Lancet 2010; 375: 895-905.

8 Buscemi S, Verga S, Batsis JA, Cottone S, Mattina A, Re A, Arnone M, Citarda S, Cerasola G. Intra-renal hemodynamics and carotid intima-media thickness in the metabolic syndrome. Diabetes Res Clin Pract 2009; 86: 177-185.

9 Florczak E, Januszewicz M, Januszewicz A, Prejbisz A, Kaczmarska M, Michalowska I, Kabat M, Rywik T, Rynkun D, Zielinski T, Kusmierczyk-Droszcz B, Pregowska-Chwala B, Kowalewski G, Hoffman P. Relationship between renal resistive index and early target organ damage in patients with never-treated essential hypertension. Blood Press 2009; 18: 55-61.

10 Raff U, Schmidt BM, Schwab J, Schwarz TK, Achenbach S, Bar I, Schmieder RE. Renal resistive index in addition to low-grade albuminuria complements screening for target organ damage in therapy-resistant hypertension. J Hypertens 2010; 28: 608-614.

11 Fukuda M, Munemura M, Usami T, Nakao N, Takeuchi O, Kamiya Y, Yoshida A, Kimura G. Nocturnal blood pressure is elevated with natriuresis and proteinuria as renal function deteriorates in nephropathy. Kidney Int 2004; 65: 621-625.

12 Petersen LJ, Petersen JR, Talleruphuus U, Ladefoged SD, Mehlsen J, Jensen HA The pulsatility index and the resistive index in renal arteries. Associations with long-term progression in chronic renal failure. Nephrol Dial Transplant 1997; 12: $1376-1380$.

13 Platt JF, Ellis JH, Rubin JM, Merion RM, Lucey MR. Renal duplex Doppler ultrasonography: a noninvasive predictor of kidney dysfunction and hepatorenal failure in liver disease. Hepatology 1994; 20: 362-369.

14 Platt JF, Rubin JM, Ellis JH. Lupus nephritis: predictive value of conventional and Doppler US and comparison with serologic and biopsy parameters. Radiology 1997; 203: 82-86.

15 Radermacher J, Ellis S, Haller $\mathrm{H}$. Renal resistance index and progression of renal disease. Hypertension 2002; 39 (Part 2): 699-703.

16 Splendiani G, Parolini C, Fortunato L, Sturniolo A, Costanzi S. Resistive index in chronic nephropathies: predictive value of renal outcome. Clin Nephrol 2002; 57: 45-50.

17 Kawai T, Kamide K, Onishi M, Yamamoto-Hanasaki H, Baba Y, Hongyo K, Shimaoka I, Tatara Y, Takeya Y, Ohishi M, Rakugi H. Usefulness of the resistive index in renal Doppler ultrasonography as an indicator of vascular damage in patients with risks of atherosclerosis. Nephrol Dial Transplant; advance access published 3 March 2011, doi:10.1093/ndt/gfr054.

18 Schwaighofer B, Kainberger F, Fruehwald F, Huebsch P, Gritzmann N, Karnel F, Tscholakoff D. Duplex sonography of normal renal allografts. Acta Radiol 1989; 30 . 53-56.

19 London NJ, Aldoori MI, Lodge VG, Bates JA, Irving HC, Giles GR. Reproducibility of Doppler ultrasound measurement of resistance index in renal allografts. $\mathrm{Br} J$ Radiol 1993; 66: 510-513

20 Knapp R, Plötzeneder A, Frauscher F, Helweg G, Judmaier W, zur Nedden D, Recheis W, Bartsch G. Variability of Doppler parameters in the healthy kidney: an anatomicphysiologic correlation. J Ultrasound Med 1995; 14: 427-429.

21 Mikkonen RH, Kreula JM, Virkkunen PJ. Reproducibility of Doppler ultrasound measurements. Acta Radiol 1996; 37: 545-550.

22 Matsuo S, Imai E, Horio M, Yasuda Y, Tomita K, Nitta K, Yamagata K, Tomino Y, Yokoyama $\mathrm{H}$, Hishida A. Revised equations for estimated GFR from serum creatinine in Japan. Am J Kidney Dis 2009; 53: 982-992.

23 American Diabetes Association Clinical Practice Recommendations 2001.. Diabetes Care 2001; 24 (Suppl 1): S1-S133.

24 Muntner P, Shimbo D, Tonelli M, Reynolds K, Arnett DK, Oparil S. The relationship between visit-to-visit variability in systolic blood pressure and all-cause mortality in the general population: findings from III NHANES, 1988 to 1994 . Hypertension 2011; 57: 160-166.

25 Ikee R, Kobayashi S, Hemmi N, Imakiire T, Kikuchi Y, Moriya H, Suzuki S, Miura S. Correlation between the resistive index by Doppler ultrasound and kidney function and histology. Am J Kidney Dis 2005; 46: 603-609.

26 Kilpatrick ES, Rigby AS, Atkin SL. The role of blood pressure variability in the development of nephropathy in type 1 diabetes. Diabetes Care 2010; 33: 2442-2447.

27 Rothwell PM, Howard SC, Dolan E, O'Brien E, Dobson JE, Dahlof B, Poulter NR, Sever PS. Effects of beta blockers and calcium-channel blockers on within-individual variability in blood pressure and risk of stroke. Lancet Neurol 2010; 9: 469-480.

28 Folkow B. Structure and function of the arteries in hypertension. Am Heart J 1987; 114 (Part 2): 938-948.

29 Franklin SS, Gustin Wt, Wong ND, Larson MG, Weber MA, Kannel WB, Levy D. Hemodynamic patterns of age-related changes in blood pressure. The Framingham Heart Study. Circulation 1997; 96: 308-315. 